\title{
Genetic investigation of multicentric glioblastoma multiforme: case report
}

\author{
*Brett Schroeder, MS, ${ }^{1,2}$ Nameeta Shah, PhD, ${ }^{2}$ Steve Rostad, MD, ${ }^{3}$ Brendan McCullough, MD, PhD, ${ }^{4}$ \\ Brian Aguedan, RT, ${ }^{4}$ Greg Foltz, MD, ${ }^{2}$ and Charles Cobbs, MD $^{2}$ \\ ${ }^{1}$ Michigan State University College of Medicine, Grand Rapids, Michigan; ${ }^{2}$ Center for Advanced Brain Tumor Treatment, Swedish \\ Neuroscience Institute; ${ }^{3}$ CellNetix Pathology \& Laboratories, Seattle; and ${ }^{4}$ Department of Radiology, Radia Inc., Everett, \\ Washington
}

\begin{abstract}
The authors report a case of multicentric glioblastoma multiforme (GBM) in which all 4 tumor foci were resected and evaluated using both comparative genomic hybridization array and RNA sequencing. Genetic analysis showed that the tumors shared a common origin, although each had its own unique set of genetic aberrations. The authors note that the genetic heterogeneity of multicentric GBM likely contributes to the failures of current treatments. The case underscores the necessity of increased genetic investigation.
\end{abstract}

http://thejns.org/doi/abs/10.3171/2015.4.JNS142231

KEY WORDS GBM; multicentric; RNA-sequencing; brain tumor; CGH array; oncology

$\mathrm{G}$ LIOBLASTOMA multiforme (GBM), a Grade IV astrocytoma, is the most malignant and most common primary brain tumor. ${ }^{8}$ Recent studies suggest that nearly $80 \%$ of all malignant brain tumors are gliomas, and 54\% of all malignant brain tumors are GBMs. ${ }^{4}$ The standard of care for GBM is resection followed by adjuvant radiation and chemotherapy, yet median survival times remain poor. ${ }^{14}$ The prognosis is worse for patients with multiple lesions than for those with a single focuswith a median overall survival of 10 versus 18 months in one recent single-institution retrospective study. ${ }^{15}$

Systematic studies analyzing GBM genomics demonstrate substantial intertumoral heterogeneity. ${ }^{2,3,10}$ There is also increasing evidence to suggest that GBM tumors have considerable intratumoral heterogeneity and are made up of genetically unique subclones that can evade standard therapy modalities, resulting in disease progression. ${ }^{6,9}$ Furthermore, a recent study found a high level of cell-to-cell diversity within individual GBM tumors. ${ }^{11}$

\section{Case Report}

History and Examination

This 47-year-old man presented to an outside clinic with leg weakness, near falls, and left inferior quadrantanopia, as well as progressive right-sided headache that primarily occurred upon standing. His medical history was significant for a lumbar laminectomy for spinal stenosis. The initial workup included a CT scan, which revealed 3 right-hemisphere lesions, leading to the patient's transfer to our neurosurgical clinic. Neurological examination revealed no confusion, no problems with memory, and no language-associated problems. Based on these findings, a brain MRI was ordered.

On MRI, 3 enhancing mass lesions were identified along with hippocampal involvement (Fig. 1). The masses were in the right frontal lobe $(2.6 \times 2.5 \times 4.4 \mathrm{~cm})$, right occipital horn region $(2.1 \times 3.6 \times 2.0 \mathrm{~cm})$, and right temporal lobe $(2.7 \times 4.1 \times 2.9 \mathrm{~cm})$. The relative cerebral blood volume (rCBV) was variably elevated for these lesions, measuring 3.1, 3.0, and 3.1 times that of normal-appearing, contralateral white matter, respectively, as measured by first-pass dynamic contrast enhancement (DCE). Each of these lesions demonstrated marked surrounding hyperintensity on FLAIR sequences. Additionally, there was a fourth nonenhancing, FLAIR-hyperintense mass lesion involving the right hippocampus $(\mathrm{rCBV}=2.1)$. The FLAIR sequences

ABBREVIATIONS $\mathrm{CGH}$ = comparative genomic hybridization; $\mathrm{DCE}=$ dynamic contrast enhancement; $\mathrm{GBM}=$ glioblastoma multiforme; rCBV = relative cerebral blood volume.

SUBMITTED September 30, 2014. ACCEPTED April 8, 2015.

INCLUDE WHEN CITING Published online October 16, 2015; DOI: 10.3171/2015.4.JNS142231.

* Mr. Schroeder and Dr. Shah contributed equally to this work. 

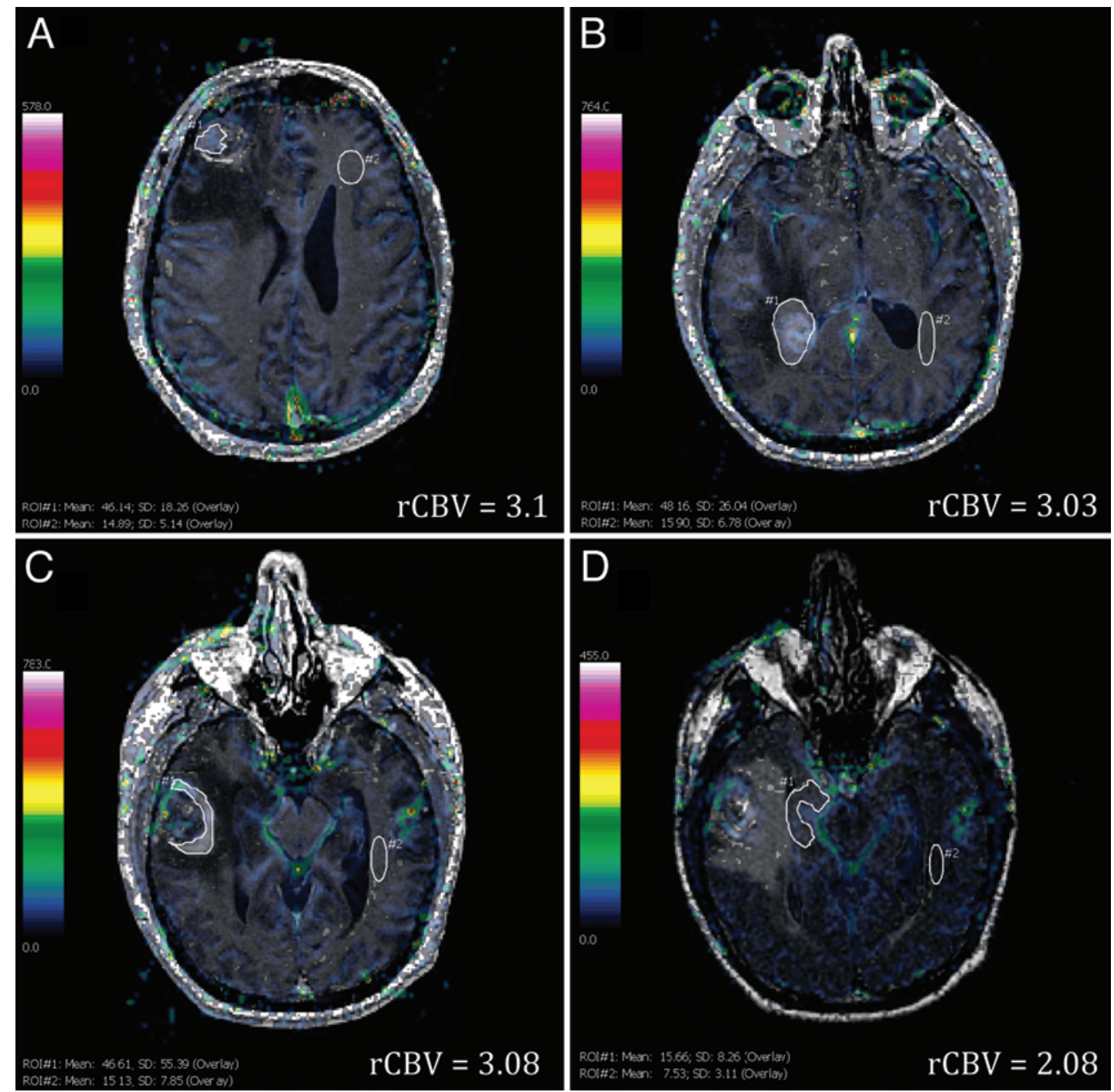

FIG. 1. Preoperative MR images with first-pass dynamic contrast enhancement (DCE). Relative cerebral blood volume (rCBV) was variably elevated for these lesions, versus that of normal-appearing, contralateral white matter as measured by first-pass DCE. A: Right frontal lobe. B: Right occipital horn region. C: Right temporal lobe. D: Right hippocampus. Figure is available in color online only.

showed that the lesion in the right frontal lobe was well separated from the others. FLAIR imaging did not show evidence of tracts connecting the tumors, and enhancing lesions had hyperintensity that radiated away from them (infiltrative edema) with minimal overlap. The appearance was not one of unidirectional extension of FLAIR hyperintensity with multifocal enhancing components, or gliomatosis cerebri.

\section{Operation}

The patient underwent craniotomies for removal of tumors in the right frontal lobe, right occipital horn region, right temporal lobe, and hippocampus. Intraoperatively, all of the tumors appeared grossly abnormal-grayish and hypervascular-and complete resections were achieved. Specimens were sent to pathology with frozen sections confirming GBM for each lesion. The remaining samples were sent to the laboratory for genetic analysis and cryogenic preservation.

\section{Histological Evaluation}

Histopathological examination (Fig. 2) of each sample using H\&E stain showed brain tissue with a highly cellular pleomorphic astrocytic neoplasm. Microvascular proliferation and significant necrosis was present in each sample except for those from the hippocampal lesion. The histological diagnosis for each of the specimens was consistent with GBM.

\section{Postoperative Treatment}

The patient underwent adjuvant radiotherapy (56 Gy over 6 weeks) and adjuvant chemotherapy with temozolomide, which was initially administered concomitantly with radiotherapy $\left(75 \mathrm{mg} / \mathrm{m}^{2} /\right.$ day $)$ and then sequentially 


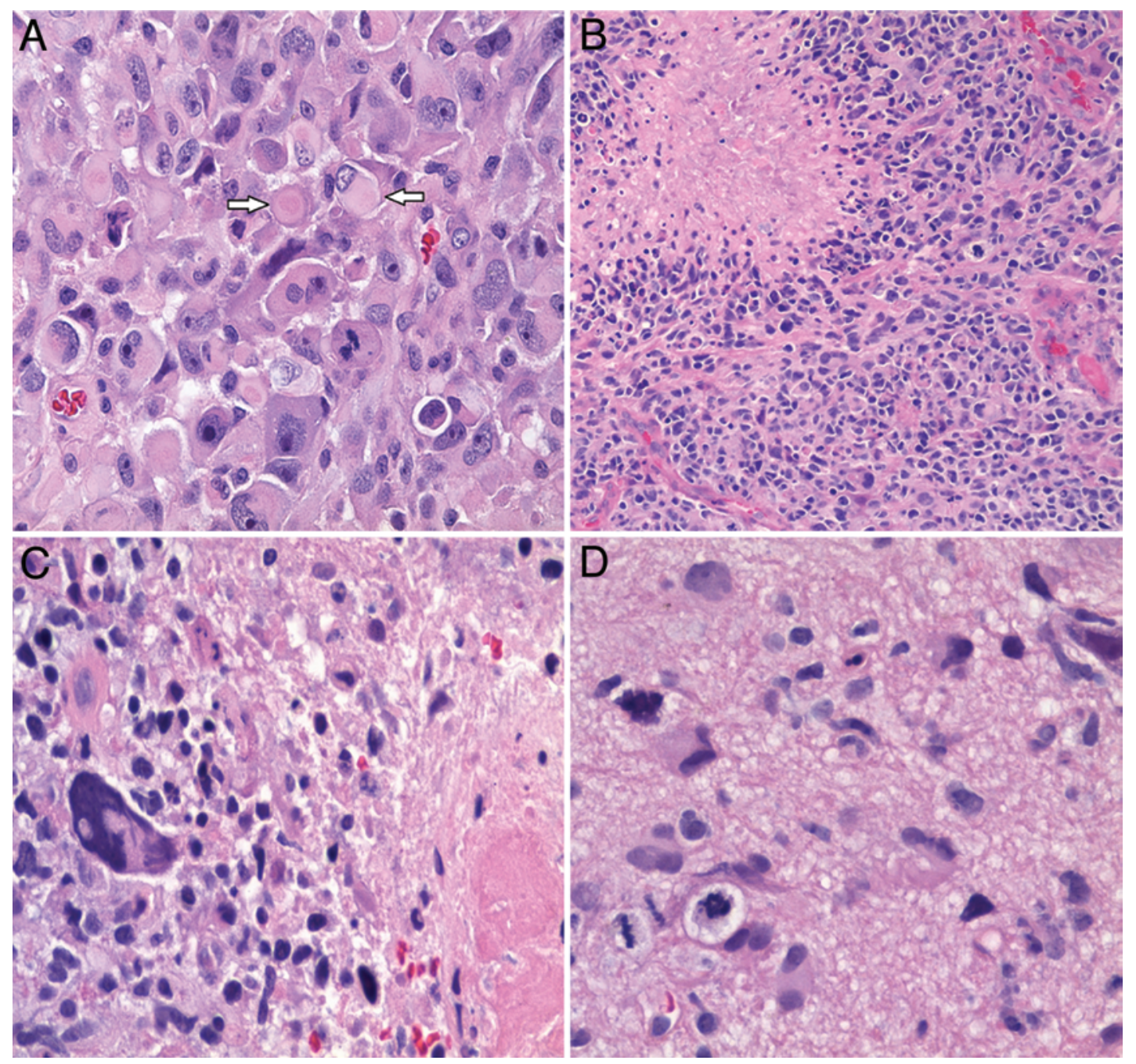

FIG. 2. Photomicrographs of $\mathrm{H} \&$ E-stained sections. A: Section from the frontal lobe tumor showing a region of moderate-sized tumor cells, some displaying rounded eosinophilic cytoplasmic contents that compress the nucleus to the periphery of the cell, commonly referred to as rhabdoid cells (arrows). B: Section from the occipital horn tumor showing cellular glioma with pseudopalisading around necrosis and microvascular proliferation. The tumor cells show nuclear hyperchromasia and pleomorphism with moderate amount of eosinophilic cytoplasm. C: Section from the temporal lobe tumor showing necrosis surrounded by cellular glioma displaying small to occasionally giant cells and marked nuclear hyperchromasia and pleomorphism. D: Section from the hippocampal lesion showing a region of moderate cellular glioma with nuclear pleomorphism, mitotic activity, and scant to moderate amounts of eosinophilic cytoplasm. Original magnification $\times 400(A, C$, and D) and 100 (B). Figure is available in color online only.

$\left(150 \mathrm{mg} / \mathrm{m}^{2} /\right.$ day for 5 days in each cycle of 28 days, for 3 cycles). The patient subsequently underwent chemotherapy with the administration of temozolomide $(75 \mathrm{mg}$ / $\mathrm{m}^{2} /$ day in each cycle of 21 days) along with bevacizumab $(10 \mathrm{mg} / \mathrm{kg}$ once every 14 days) for 6 months. The patient showed clear signs of clinical deterioration at 10 months and succumbed to the disease at 12 months.

\section{CGH Array and RNA Sequencing}

To interrogate copy number aberrations, gene expression similarities and differences, and to explore a possible lineage among the four tumors, comparative genomic hybridization ( $\mathrm{CGH})$ array $(\mathrm{aCGH})$ and transcriptome sequencing (RNA-sequencing) were performed. We used the circular binary segmentation (CBS) algorithm to cal- culate copy number changes in aCGH data. Reads from RNA-sequencing data were aligned to hg19 (Human Genome version 19), and the assembly and expression values were calculated as RPKM (reads per kilobase per million mapped reads) values using bioscope 1.3 software by Life Technologies as described. ${ }^{13}$ Hierarchical clustering of 840 genes was performed using $\mathrm{MeV}$ 4.9.0. ${ }^{12}$ The 840 genes were used to determine the molecular subtype of GBM. $^{16}$ The expression profiles were normalized using standard score and then a pairwise average-linkage clustering method was used and visualized in MeV (Fig. 3). As shown, frontal, temporal, and occipital horn tumor samples (SN132_1, SN132_2, SN132_4, respectively) clustered adjacently. The hippocampal tumor (SN132_3) expression profile was distantly related and showed greater similarity 


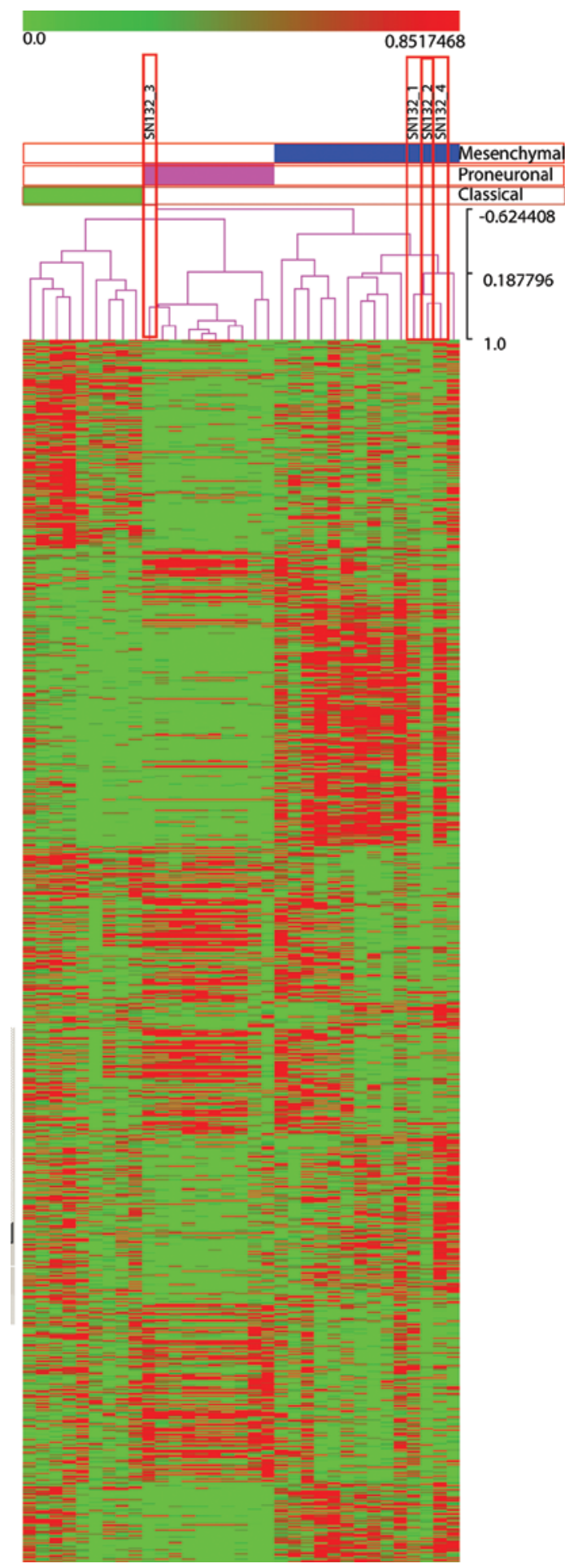

FIG. 3. Pairwise average-linkage clustering of RNA-sequencing data. Hierarchical clustering of 840 gene expression profiles from RNA-sequencing data (28 GBM and 4 nontumor brain samples) was performed using MeV 4.9.0. The 840 genes were used to determine the molecular subtype of GBM. Frontal, temporal, and occipital horn tumor samples (SN132_1, SN132_2, SN132_4, respectively) clustered adjacently as mesenchymal subtype (blue bar). The hippocampal tumor (SN132_3) expression profile was distantly related, had proneuronal subtype (magenta bar), and showed greater similarity to tumors resected from different GBM patients with proneuronal subtype. Figure is available in color online only. to tumors resected from different GBM patients. Furthermore, RNA-sequencing and aCGH expression data (Fig. 4) showed a shared set of aberrations among the hippocampal, frontal, temporal, and occipital horn lesions. All 4 tumors harbored a R175H mutation in the TP53 gene, had focal amplification on chromosome 6 accompanied by overexpression of the HDAC2 and MARCKS genes in that locus, deletion of the $C D K N 2 A / C D K N 2 B$ locus, and loss of expression of the MTSSI gene due to focal deletion on chromosome 8 . However, each of these masses also contained unique aberrations that made them highly differentiated. The hippocampal tumor had MET amplification, the frontal tumor had focal chromosome 3 amplification, the occipital tumor had focal amplification on chromosome 9 , and the temporal tumor showed focal amplification of the EGFR locus and presence of an EGFRvIII mutation. These data suggested that all of the tumors had a common origin while each had evolved a unique genetic signature. Thus, a diagnosis of multicentric GBM was made.

\section{Discussion}

Treating patients with multiple GBM lesions has been challenging for oncologists and surgeons, especially since no definitive treatment guidelines exist. The incidence of multiple and simultaneous brain cancer foci upon diagnosis has historically been reported as between $0.5 \%$ and $20 \% .{ }^{1,7}$ However, a recent study found that $35 \%$ of patients had multiple lesions at the time of diagnosis..$^{15}$ This prevalence necessitates further investigation.

Of note, the clinical strategy chosen in our case-resecting multiple lesions-is more aggressive than usual. The patient had 3 children and was in otherwise good health. Also, the resections were not performed for investigative purposes. It has been reported that the use of multiple craniotomies for multiple lesions is not associated with increased risks or complications (vs a single resection) and that the duration of survival for GBM patients with multiple lesions in which all lesions were resected was similar to that of patients in a matched cohort who underwent resection of a single glioblastoma. ${ }^{5}$

Here we present a patient with 4 GBM tumors that were shown through in-depth genetic investigation to have a small degree of similarity and a marked level of genetic heterogeneity (when compared with one another). The data suggest that the 4 tumors share a common origin, yet each acquired a unique set of aberrations. Interestingly, even though 3 of the tumors were of the mesenchymal subtype, they were no more similar than tumors of the same subtype from different patients.

In an era of personalized medicine based on technological advancements, greater genetic interrogation is needed to produce more meaningful long-term survival data, particularly in patients with multiple glioblastoma lesions. Therapeutic advances have done little to minimize disease progression or to improve survival for this subpopulation of patients. In cases of multicentric and multifocal disease, genetic investigation of each tumor may permit more precise tumor characterizations, creating highly targeted therapies to improve patient outcomes. 


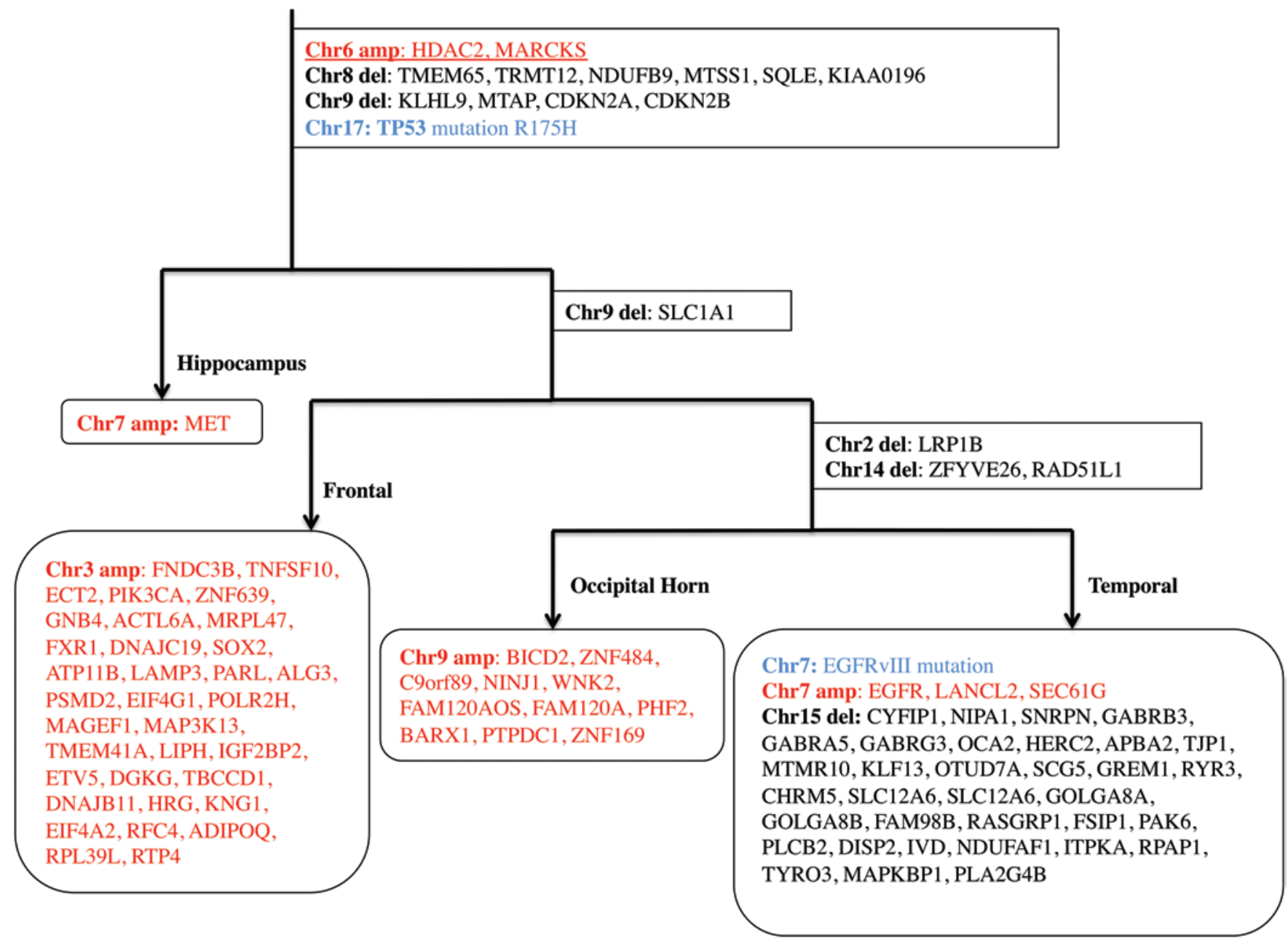

FIG. 4. Tumor lineage based on RNA-sequencing and aCGH data. All 4 tumors harbor an R175H mutation in the TP53 gene, have overexpression of the HDAC2 and MARCKS genes due to focal amplification on chromosome 6, deletion of the CDKN2A/ CDKN2B locus, and loss of expression of several genes, including MTSS1, due to focal deletion on chromosome 8. The hippocampal tumor has MET overexpression and amplification. The frontal, occipital, and temporal lobe tumors share loss of expression of SLC1A1 due to chromosomal loss. The frontal lobe tumor has focal chromosome 3 amplification accompanied by overexpression of several genes in that locus. The occipital and temporal lobe tumors share deletion of the LRP1B, ZFYVE26, and RAD51L1 genes. The occipital lobe tumor has focal amplification on chromosome 9 accompanied by overexpression of several genes in that locus, and the temporal lobe tumor shows focal amplification of the EGFR locus and presence of EGFRvIll mutation. Amp = amplification; del = deletion; $\mathrm{chr}=$ chromosome. Figure is available in color online only.

\section{Acknowledgments}

We gratefully acknowledge the Ben and Catherine Ivy Foundation and the Swedish Medical Foundation for their support of this project. We also acknowledge CODONiS for providing the computational infrastructure for bioinformatics analyses. The funders had no role in the study design, data collection and analysis, decision to publish, or preparation of the manuscript.

\section{References}

1. Barnard RO, Geddes JF: The incidence of multifocal cerebral gliomas. A histologic study of large hemisphere sections. Cancer 60:1519-1531, 1987

2. Brennan CW, Verhaak RG, McKenna A, Campos B, Noushmehr H, Salama SR, et al: The somatic genomic landscape of glioblastoma. Cell 155:462-477, 2013

3. Cancer Genome Atlas Research Network: Comprehensive genomic characterization defines human glioblastoma genes and core pathways. Nature 455:1061-1068, 2008

4. Central Brain Tumor Registry of the United States: CBTRUS Statistical Report: Primary Brain and Central Nervous System Tumors Diagnosed in the United States in 2004-2008. Hinsdale, IL: Central Brain Tumor Registry of the United States, 2012.

5. Hassaneen W, Levine NB, Suki D, Salaskar AL, de Moura Lima A, McCutcheon IE, et al: Multiple craniotomies in the management of multifocal and multicentric glioblastoma. Clinical article. J Neurosurg 114:576-584, 2011

6. Johnson BE, Mazor T, Hong C, Barnes M, Aihara K, McLean CY, et al: Mutational analysis reveals the origin and therapy-driven evolution of recurrent glioma. Science 343:189-193, 2014

7. Kyritsis AP, Levin VA, Yung WK, Leeds NE: Imaging patterns of multifocal gliomas. Eur J Radiol 16:163-170, 1993

8. Louis DN, Ohgaki H, Wiestler OD, Cavenee WK, Burger PC, 
Jouvet A, et al: The 2007 WHO classification of tumours of the central nervous system. Acta Neuropathol 114:97-109, 2007

9. Navin N, Kendall J, Troge J, Andrews P, Rodgers L, McIndoo $\mathrm{J}$, et al: Tumour evolution inferred by single-cell sequencing. Nature 472:90-94, 2011

10. Parsons DW, Jones S, Zhang X, Lin JC, Leary RJ, Angenendt $\mathrm{P}$, et al: An integrated genomic analysis of human glioblastoma multiforme. Science 321:1807-1812, 2008

11. Patel AP, Tirosh I, Trombetta JJ, Shalek AK, Gillespie SM, Wakimoto H, et al: Single-cell RNA-seq highlights intratumoral heterogeneity in primary glioblastoma. Science 344:1396-1401, 2014

12. Saeed AI, Sharov V, White J, Li J, Liang W, Bhagabati N, et al: TM4: a free, open-source system for microarray data management and analysis. Biotechniques 34:374-378, 2003

13. Shah N, Lankerovich M, Lee H, Yoon JG, Schroeder B, Foltz G: Exploration of the gene fusion landscape of glioblastoma using transcriptome sequencing and copy number data. BMC Genomics 14:818, 2013

14. Stupp R, Hegi ME, Mason WP, van den Bent MJ, Taphoorn MJ, Janzer RC, et al: Effects of radiotherapy with concomitant and adjuvant temozolomide versus radiotherapy alone on survival in glioblastoma in a randomised phase III study: 5-year analysis of the EORTC-NCIC trial. Lancet Oncol 10:459-466, 2009

15. Thomas RP, Xu LW, Lober RM, Li G, Nagpal S: The inci- dence and significance of multiple lesions in glioblastoma. $\mathbf{J}$ Neurooncol 112:91-97, 2013

16. Verhaak RG, Hoadley KA, Purdom E, Wang V, Qi Y, Wilkerson MD, et al: Integrated genomic analysis identifies clinically relevant subtypes of glioblastoma characterized by abnormalities in PDGFRA, IDH1, EGFR, and NF1. Cancer Cell 17:98-110, 2010

\section{Disclosure}

The authors report no conflict of interest concerning the materials or methods used in this study or the findings specified in this paper.

\section{Author Contributions}

Conception and design: Foltz. Acquisition of data: Shah, Rostad, McCullough, Aguedan, Foltz. Analysis and interpretation of data: Schroeder, Shah, Rostad, McCullough, Aguedan, Foltz. Drafting the article: Schroeder, Shah. Critically revising the article: Schroeder, Shah, Rostad. Reviewed submitted version of manuscript: Cobbs, Schroeder, Shah. Statistical analysis: Shah, McCullough. Administrative/technical/material support: Schroeder. Study supervision: Cobbs, Foltz.

\section{Correspondence}

Charles Cobbs, Swedish Neuroscience Institute, 550 17th Ave., Seattle, WA 98122. email: charles.cobbs@swedish.org. 